\title{
Sistemática para apuração de custos por procedimento médico-hospitalar
}

\author{
Maury Leoncine ${ }^{a *}$, Antonio Cezar Bornia ${ }^{\mathrm{b}}$, Katia Abbas ${ }^{\mathrm{c}}$ \\ a*maury_leoncine@yahoo.com.br, UFSC, Brasil

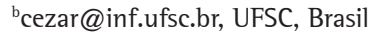 \\ ckatia_abbas@yahoo.com.br, UEM, Brasil
}

\begin{abstract}
Resumo
O objetivo deste artigo é apresentar uma sistemática para apuração de custos por procedimento médico-hospitalar e, assim, contribuir para a melhoria do gerenciamento dos resultados operacionais de instituições de saúde. Pesquisas de custos em serviços de saúde usualmente têm se concentrado na escolha e aplicação dos métodos de custeio (ABC, centros de custos), sem dar ênfase à geração de resultados para a tomada de decisão. No entanto, a implantação de um sistema de custos em instituições de saúde, além de propiciar a gestão de custos, deve também auxiliar na análise de resultados. A pesquisa pode ser classificada como aplicada, exploratória e qualitativa com uso de pequisa-ação. Foi proposta uma sistemática composta por 13 etapas sequenciais, permitindo ao hospital a apuração do resultado por procedimento médico-hospitalar. Concluiu-se que o emprego da sistemática disponibilizou ao hospital informações que possibilitam o conhecimento do custo por procedimento, além de outras informações que contribuem para a melhoria da gestão dos resultados.
\end{abstract}

Palavras-chave

Método dos centros de custos. Análise de resultados. Análise de procedimentos. Análise custo-volume-lucro. Custos hospitalares.

\section{Introdução}

0 hospital moderno é uma organização quase completa, em termos da área da saúde. Sua equipe congrega profissionais médicos, paramédicos e administradores qualificados e incorpora o avanço constante dos conhecimentos, das aptidões, das tecnologias da medicina e dos aspectos finais dessas tecnologias representados pelas instalações e equipamentos (COLTRO; SANTOS, 1998).

Os usuários que procuram um serviço no hospital são na maioria das vezes originários de um encaminhamento médico para um determinado procedimento (por exemplo: uma cirurgia de hérniorrafia, uma revascularização do miocárdio, um parto cesárea). Os casos de urgência são originários do próprio serviço de pronto socorro do hospital e há também usuários originários de tratamento de longo prazo feito em consultório. Portanto, os pacientes chegam ao hospital em busca de um tratamento - conhecido como procedimento médico - e não simplesmente para utilizar um serviço (uma diária, uma hora de centro cirúrgico ou uma refeição, por exemplo).

A gestão de custos em hospitais é um importante instrumento de gerenciamento e controle dos custos incorridos nos centros de custos. A literatura apresenta vários métodos de custeio, porém, o método dos centros de custos, segundo Matos (2002), é o mais viável de ser implantado no hospital como um todo, pela complexidade, pelas informaç̧ões e controles gerados.

Cita ainda o referido autor que o método dos centros de custos representa o instrumento mais tradicional de gestão de custos. Assim, essa abordagem de custeio é largamente utilizada para o processo de apuração dos custos hospitalares, ou seja, para o cálculo dos custos de todos os centros de custos, bem como para o cálculo dos custos por procedimento hospitalar. 
Segundo Matos (2002, p. 97-98):

[...] a apropriação de custos por centros de custos e o custeio por procedimento hospitalar representam as duas formas mais usuais de expressão do custo de um serviço gerado por uma empresa hospitalar. 0 custo gerado pela apropriação dos centros de custos corresponde às unidades de serviços produzidas em cada um dos centros de custos definidos para o hospital. As expressões de custo unitário associadas a cada um dos centros de custos corresponderão, portanto, a uma diária hospitalar, a uma taxa de sala cirúrgica, a uma consulta, a um exame, entre outros. 0 custo dos procedimentos hospitalares corresponde a uma sequência de cálculos, os quais compreenderão os custos unitários gerados pelos centros de custos combinados com a intensidade dos referidos insumos.

Assim, pode-se dizer que o método dos centros de custos é um eficiente sistema orientado para as decisões operacionais. Por outro lado, ele não revela alguns custos que a moderna competição global colocou em evidência. A utilização desse método deve estar aliada ao custeio variável que, segundo Souza e Clemente (2007), é um princípio que destaca o peso da estrutura organizacional e produtiva da empresa e força o confronto entre a capacidade instalada e o nível de atividade, orientando a elaboração de estratégias que buscam otimizar o uso da estrutura. Para tanto, são utilizados parâmetros, indicadores e ferramentas gerenciais, destacando-se entre esses o custo variável unitário e a margem de contribuição.

Diz Maher (2001) que na busca do equilíbrio entre receitas, custos e despesas, métodos como o custeio variável e a análise de custo-volume-lucro (CVL), largamente utilizados no setor industrial, são trazidos para a realidade de empresas de serviços. Outra vantagem da análise CVL é evitar distorções dos custos fixos indiretos, as quais sempre envolvem alguma subjetividade.

A partir da informação dos custos dos centros de custos, é possível evoluir e obter também a receita por centros de custos. Isso possibilita ao hospital obter sua margem de contribuição e seu ponto de equilíbrio e, consequentemente, efetuar a análise custo-volume-lucro por centro de custos.

Embora a conceituação e a correspondente obtenção dos custos de bens ou serviços gerados sob a classificação dos centros de custos compreenda valiosa informação de custeio das atividades de um hospital, somente a complementação com o custeio de procedimentos hospitalares encerra o ciclo da apropriação de custos sob a orientação da abordagem do método dos centros de custos (MATOS, 2002).

Dessa forma, o custo por procedimento médico-hospitalar se constitui em informação estratégica, podendo ser utilizado para a definição de alguns indicadores como: do procedimento/ convênio/médico ou especialidade com maior margem de contribuição, proporcionando assim informações mais adequadas para o gerenciamento da informação no hospital. Entretanto, não se verifica, na prática, a utilização da informação dessa forma, havendo uma lacuna a ser preenchida pelo cálculo do custo por procedimento médico-hospitalar, analisando os que proporcionam melhor margem de contribuição.

Em face do exposto, o objetivo deste trabalho é apresentar uma sistemática que permita a apuração de custos por procedimento médico-hospitalar. Tal informação permitirá uma melhoria na gestão dos resultados operacionais de instituições de saúde.

Na próxima seção apresentam-se os conceitos teóricos utilizados para embasar a proposta, bem como trabalhos correlatos encontrados na literatura. A seção 3 esclarece a classificação da pesquisa e os procedimentos metodológicos. Após, define-se a sistemática proposta, a qual é aplicada em um hospital e, finalmente, apresentam-se as conclusões do artigo.

\section{Gestão de custos em instituições de saúde}

Segundo Bruger et al. (1997), a indústria de cuidados à saúde cresceu muito, não somente nos Estados Unidos mas também em outros países. Porém, os interessados, como o governo, as financiadoras e mesmo alguns grupos de consumidores estão preocupados com o aumento crescente dos gastos vinculados à saúde e o seu aparentemente baixo nível de eficácia.

Apesar desses gastos com a saúde, parece não haver muitos hospitais no Brasil que cobram suas taxas com embasamento técnico, ou seja, os custos dos procedimentos são desconhecidos. Baumgartner (1998) já dizia que a era do empirismo na gestão hospitalar estava com os dias contados. As ferramentas administrativas e financeiras são cada vez mais necessárias. Dentre elas, a questão dos custos hospitalares é de grande importância.

Assim, o registro e a gestão estratégica de custos hospitalares são necessários nas instituições hospitalares, visto que o registro serve de instrumento eficaz de gerência e acompanhamento dos serviços, enquanto que a gestão estratégica de custos permite a implantação de medidas corretivas que visem a um melhor desempenho das unidades, com base na possível redefinição das prioridades essenciais, aumento da produtividade e racionalização do uso de recursos, dentre outras medidas administrativas (OLIVEIRA et al., 2007). 
A margem de contribuição de um serviço prestado é a diferença entre o valor das receitas e os custos variáveis totais. Assim, essa ferramenta permite avaliar o quanto cada serviço contribui na amortização dos custos fixos e das despesas fixas e na geração do lucro. Segundo Falk (2001), Matos (2002), Souza e Clemente (2007), Bornia (2009), Perez Junior, Oliveira e Costa (2009), Ribeiro (2009) e Santos (2009), o ponto de equilíbrio representa uma importante ferramenta de gestão de resultados operacionais da empresa. Ele expressa o nível de atividades em que o resultado operacional da empresa é zero, em que momento ou contexto ocorre, portanto, a igualdade entre receita total e o custo total.

0 método do centro de custos, criado na Europa no início do século XX, é o mais utilizado no mundo (BORNIA, 2009; PEREZ JUNIOR; OLIVEIRA; COSTA, 2009). Segundo Matos (2002), é o mais viável, considerando a realidade dos hospitais, por disponibilizar informações sobre o quanto foi gasto e indicar a quais centros de custos se vinculam os gastos do hospital.

Muitas das pesquisas e debates sobre custos têm se concentrado na aplicação dos métodos de custeio sem dar a devida importância à utilização do método para gerar resultados para a tomada de decisão. Além disso, em sua maior parte, as pesquisas estão focadas no custeio baseado em atividades, em aplicações restritas a uma unidade do hospital, apresentando-se o método dos centros de custos como o mais adequado para fins de gestão e tomada de decisão.

Baumgartner (1998) defendeu a utilização do ABC (custeio baseado em atividades) e desenvolveu um caso prático apurando o custo paciente-dia da UTI (unidade de tratamento intensivo) de um hospital de emergência tanto pelo método dos centros de custos quanto pelo de custeio baseado em atividades.

Abbas (2001) defendeu a utilização do ABC para o hospital e o utilizou para o cálculo do quilograma da roupa lavada no setor de processamento de roupas de um hospital de ensino.

Vargas (2002) aplicou o ABC em três procedimentos específicos de um hospital: colecistectomia, revascularização do miocárdio e endardectomia, sugerindo sua utilização em todos os setores do hospital.

Martins (2002) propôs a construção de um modelo de gestão de resultados para serviços hospitalares. Esse modelo apoiou-se na gestão de processos, utilizando o $A B C$ para custear as atividades e os objetos de custos.

Rocha (2004) identificou as dificuldades na aplicação tanto do método dos centros de custos quanto do $A B C$ e enfatizou a impossibilidade da aplicação do $A B C$ devido às próprias características do setor hospitalar. Para a referida autora, o método dos centros de custos são mais eficazes para os hospitais.

Souza (2004) realizou um estudo comparativo e constatou que o método de centros de custos utilizado no hospital é adequado. Sua pesquisa abrangeu a apuração do custo de alguns procedimentos.

Raimundini et al. (2006) aplicaram o ABC no setor de ginecologia e obstetrícia de dois hospitais públicos com o objetivo de obter os custos dos procedimentos parto normal e cesárea e verificar se o custo desse é inferior ou não aos repasses feitos pelo SUS. Também foi apresentada a margem de contribuição de cada procedimento de parto em cada um dos hospitais.

Silva (2006) sugeriu a aplicação de um método de custeio híbrido utilizando o $\mathrm{ABC}$ com o método da unidade de esforço de produção para o custeamento dos procedimentos relacionados ao parto no setor de obstetrícia.

Schultz (2007) apurou o custo através do método de centros de custos e incorreu na análise de resultado apenas no serviço de hemodinâmica do hospital.

Souza et al. (2008) defenderam a aplicação do ABC, apresentando uma aplicação no setor de centro cirúrgico de três hospitais privados da cidade de Belo Horizonte, Minas Gerais.

0 Quadro 1 sintetiza o método de custeio utilizado pelos referidos autores. Nota-se que o $A B C$ é o mais defendido pelos autores, com a justificativa de que o método proporciona informações mais precisas. Porém a adoção do método para o hospital todo, devido às próprias características do setor hospitalar, envolveria muitos recursos. 0 levantamento de todas as atividades do hospital seria inviável também sob o ponto de vista financeiro. É possível sim, a aplicação em uma unidade, como, por exemplo, o centro cirúrgico ou a lavanderia. $\mathrm{O} A \mathrm{BC}$ pode ser usado como um complemento do método dos centros de custos, auxiliando na obtenção de melhores informações de setores ou processos que se mostram mais problemáticos.

Quadro 1. Método de custeio utilizado (elaborado pelos autores).

\begin{tabular}{ccc}
\hline \multirow{2}{*}{ Autores } & \multicolumn{2}{c}{ Método de custeio } \\
\cline { 2 - 3 } & Centros de custos & $\mathrm{ABC}$ \\
\hline Baumgartner (1998) & & $\mathrm{X}$ \\
Abbas (2001) & $\mathrm{X}$ \\
Vargas (2002) & $\mathrm{X}$ & $\mathrm{X}$ \\
Martins (2002) & $\mathrm{X}$ & \\
Rocha (2004) & & \\
Souza (2004) & & $\mathrm{X}$ \\
Raimundini et al. (2006) & $\mathrm{X}$ & $\mathrm{X}$ \\
Silva (2006) & & $\mathrm{X}$ \\
Schultz (2007) & & \\
Souza et al. (2008) & & \\
\hline
\end{tabular}


Os autores que defendem a utilização do método dos centros de custos, como Rocha (2004) e Schultz (2007), mostram a aplicação do método sem, contudo, demonstrar a efetiva utilização dos custos nas análises do resultado financeiro do hospital auxiliando a administração na tomada de decisões. Suas aplicações se restringem a apenas alguns procedimentos do hospital e não a todos.

Para Oliveira et al. (2007), o nível de apuração das informações de custos também implica em resultados muito diversos no que diz respeito à gestão dos hospitais. Em muitas instituições hospitalares, os sistemas de custeio implantados restringem-se à apuração dos custos setoriais e, em alguns casos, atendem a análises de avaliação de resultado por área (BEULKE; BERTÓ, 1997).

Porém, ainda que esses hospitais estejam evoluídos em relação a outros, uma etapa importante, que é a apuração do custo dos procedimentos hospitalares com a atribuição dos custos das áreas ou atividades de cada serviço prestado, deixa de ser cumprida.

Diante do exposto, é viável a utilização do método dos centros de custos com a acumulação de custos por procedimento hospitalar.

\subsection{Classificação da pesquisa e procedimentos metodológicos}

Empregando a classificação proposta por Silva e Menezes (2001), sob ponto de vista da sua natureza, esta pesquisa pode ser classificada como aplicada. Ela aborda o problema de forma qualitativa, com uso da pesquisa-ação como procedimento técnico. Quanto aos objetivos, pode ser classificada como exploratória, pois objetiva proporcionar maior familiaridade com o problema, com vistas a torná-lo explícito.

Foi elaborada uma sistemática para apuração de custos por procedimento médico-hospitalar a qual foi aplicada em um hospital privado de médio porte da cidade de Campo Grande, Mato Grosso do Sul. A escolha do referido hospital se deveu à necessidade dos gestores do hospital de calcular o custo dos procedimentos médico-hospitalares, pois pretendiam com esses dados melhorar o gerenciamento dos resultados operacionais.

A coleta de dados foi realizada no período de maio a agosto de 2009, principalmente no departamento de controladoria do hospital. Após, os dados foram trabalhados com o intuito de se obter o custo por procedimento. Ressalta-se que um dos autores do trabalho esteve diretamente envolvido em cada uma das etapas da aplicação.

\subsection{Sistemática aplicada}

A Figura 1 apresenta uma visão geral da sistemática, a qual está estruturada em 13 etapas que devem ser realizadas de forma sequenciada.

Para a elaboração da sistemática foram consideradas as técnicas e os conceitos apresentados na literatura.

A etapa 1 consiste em determinar as pessoas responsáveis pela implementação da sistemática. É importante que na equipe haja no mínimo a participação de uma pessoa da área financeira e que haja apoio da direção do hospital. Na etapa 2 sugere-se que seja feita uma reunião para apresentação da sistemática, esclarecimento das etapas, exposição de conceitos e metodologias a serem aplicadas e conscientização das pessoas envolvidas. A etapa 3 compreende a identificação da estrutura física do hospital, com o detalhamento das principais características dos setores, tais como tamanho, número de funcionários, quantidade de equipamentos e atividades desenvolvidas. Na etapa 4 , reuniões individuais com os responsáveis pelas unidades objetivam validar a característica de cada setor e avaliar a possibilidade de abertura de centros de custos com a respectiva definição da base de distribuição e/ou unidade de produção. A etapa 5 consiste em um diagnóstico do sistema de gestão hospitalar, com a identificação de características do sistema de gestão (disponibilidade de dados, abrangência da informação, verificação dos setores que utilizam o sistema de gestão) e a busca de informações criteriosas por meio de dados disponíveis no sistema de gestão, bem como uma análise criteriosa do módulo financeiro/contábil para classificação dos itens de custos pela facilidade de alocação (direto ou indireto) e pela variabilidade (fixo ou variável). Na etapa 6 , define-se a estrutura dos centros de custos, com auxílio da avaliação do sistema de gestão hospitalar, do organograma, bem como com base nas reuniões com os responsáveis pelos setores, considerando a necessidade de controle sugerido pela instituição e considerando a disponibilidade de recursos e estatísticas. Na etapa 7 são elaborados os formulários de coleta de dados, pois alguns dados não podem ser obtidos através do sistema de gestão. Nesse caso é preciso aprimoramento do sistema de gestão ou o estabelecimento de formas de controle manual para a coleta de dados. Na etapa 8 é definida a forma como os dados serão processados, ou seja, é desenvolvido o aplicativo de informática. Deve ser verificada a disponibilidade de sistema informatizado que esteja dentro dos conceitos apresentados para apuração dos custos. Na etapa 9 processam-se os dados e faz-se a execução da apuração do custo por centro de custos. Depois de estruturado o sistema de custos com seus respectivos cadastros e parametrizações, o sistema efetua os cálculos necessários para apuração dos custos por centro de custos. Na etapa 10 é feita 


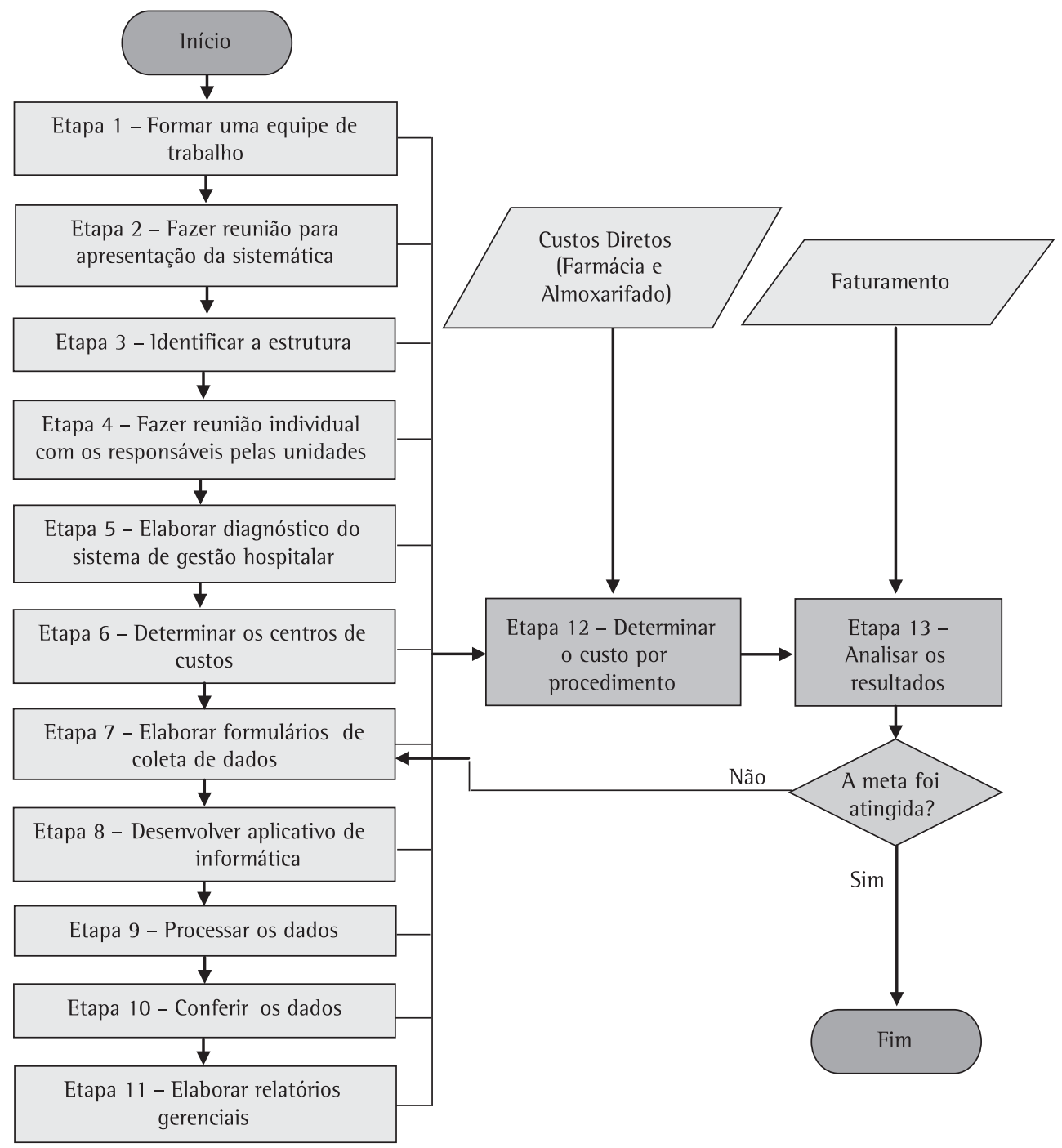

Figura 1. Sistemática para apuração de custos por procedimento médico-hospitalar.

uma conferência de dados, ou seja, uma verificação e análise da apuração dos custos por centro de custos com intuito de detectar possíveis erros de lançamento. $\mathrm{Na}$ etapa 11 devem ser elaborados os relatórios gerenciais que darão suporte à análise e acompanhamento mensal da apuração dos custos por centro de custos. Na etapa 12 é feita a apuração do custo por procedimento com a identificação dos itens lançados em cada conta hospitalar (conta do paciente) e o custo de cada item calculado pelo sistema de custos. Por fim, a etapa 13 consiste em analisar os resultados dos procedimentos médico-hospitalares, Com isso, obtêm-se diversas análises, dentre elas a margem de contribuição, resultado que acumulando várias contas hospitalares pode proporcionar diferentes níveis de análise, como por tipo de procedimento, por médico e por convênio.
Ressalta-se que devem haver revisões periódicas no sistema, a fim de evitar a sua defasagem. Idealmente, sempre que houver modificação no ambiente o sistema deve ser atualizado.

\section{Aplicação}

O Hospital do Coração de Mato Grosso do Sul - Clínica Campo Grande concordou em aplicar a sistemática proposta pelos autores, visto que pretende aprimorar a gestão com a visão do custo por procedimento, uma vez que a apuração dos custos limita-se à informação de custos por centro de custos. Com isso, a administração desse hospital estaria com os dados validados e caminharia para um nível melhor de informação (da apuração do custo 
por centro de custo para a apuração do custo por procedimento), tendo assim melhores condições para a análise e a tomada de decisão.

0 custo do procedimento é obtido por meio do método dos centros de custos e dos lançamentos dos custos diretos feitos pela farmácia e pelo almoxarifado. Com o lançamento do faturamento na conta do paciente é possivel incorrer na análise de resultados dos procedimentos juntando-se todos os procedimentos realizados, e essa análise pode ser ampliada, incluindo a margem de contribuição, análise por convênio, por médico e por especialidade.

$\mathrm{Na}$ etapa 1 foi formada uma equipe de trabalho composta por um dos autores, por uma profissional da controladoria e pelo diretor do hospital.

$\mathrm{Na}$ etapa 2 o pesquisador apresentou à profissional de controladoria e ao diretor do hospital a sistemática proposta para apuração do custo. Nessa apresentação foram ressaltados os conceitos e as etapas a serem seguidas na sistemática. Logo após foi realizada outra reunião entre a equipe de trabalho e os gestores de cada área, quando foram apresentados novamente os conceitos, a terminologia e as etapas a serem cumpridas na sistemática.

$\mathrm{Na}$ etapa 3, a equipe de trabalho realizou uma visita física para apresentar ao pesquisador as instalações do hospital.

Na etapa 4 foram feitas reuniões individuais, dirigidas pelo pesquisador e pela profissional de controladoria, com os responsáveis por cada unidade, nas quais foram discutidos assuntos pertinentes à sistemática a ser implantada, reafirmados os conceitos apresentados na reunião geral (etapa 2) e definida a possibilidade de abertura de centro de custos com a respectiva base de rateio e/ou unidade de produção.

$\mathrm{Na}$ etapa 5 foi feito um levantamento sobre o funcionamento do sistema de gestão hospitalar utilizado. 0 hospital possuía um software de gestão hospitalar que englobava vários módulos, dentre eles: gestão clínica (ambulatório e marcação de consultas, pronto socorro, internação, centro cirúrgico, laboratório, imagens, prescrição eletrônica e prontuário eletrônico), operacional (estoque, farmácia, compras, faturamento e manutenção), administrativo (financeiro e custos) e apoio à decisão. No sistema foi verificada a existência dos dados financeiros e estatísticos necessários para implantação da sistemática. Cada item de custo foi classificado quanto à facilidade de alocação (direto ou indireto), ao grupo a que pertencia (pessoal, materiais, gerais ou indireto) e à variabilidade (fixo ou variável).

$\mathrm{Na}$ etapa 6 foi determinada a estrutura de centros de custos do hospital. 0 Quadro 2 apresenta os centros de custos e sua classificação em produtivos, auxiliares e administrativos para o hospital. Consideram-se produtivos os que geram receita e se relacionam diretamente com os procedimentos ou pacientes. Consideraram-se auxiliares os centros que prestavam serviços de apoio e os centros administrativos compreendiam a administração do hospital.

$\mathrm{Na}$ etapa 7 foram elaborados os formulários para coleta de dados para os centros de custos levantados na etapa 6 , pois alguns dados não estavam disponíveis através do sistema de gestão hospitalar (etapa 5). 0 Quadro 3 apresenta os centros de custos para os quais foram elaborados formulários, bem como o tipo de informação necessária, ou seja, os indicadores de atividades com os quais se determinará o custo unitário de cada centro de custos. Os formulários são preenchidos e entregues ao setor de custos no quinto dia útil do mês subsequente.

É importante o cumprimento do prazo de entrega para que seja viabilizado o fechamento do mês, uma vez que a informação rápida proporciona maiores condições de melhoria.

$\mathrm{Na}$ etapa 8, com todos os dados organizados, o sistema de custos (software) foi disponibilizado pela mesma empresa que fornece o sistema de gestão hospitalar. Foram cadastrados e parametrizados no sistema de custos todos os dados do projeto, tais como: a estrutura de centros de custos, as bases de rateio para os centros de custos auxiliares e administrativos, as unidades de produção para os centros de custos produtivos, as bases de rateio para os custos indiretos, os grupos de custos (pessoal, materiais, gerais e indiretos), os itens de custos que compõem os grupos de custos (salários, medicamentos, energia, dentre outros), a classificação conforme à facilidade de alocação e a variabilidade de cada item de custo. Concluída esta etapa, o sistema ficou pronto para receber os dados, processar e gerar as informações através de relatórios gerenciais.

$\mathrm{Na}$ etapa 9 foi feito o processamento dos dados com o auxílio do software no momento em que todos os dados já estavam digitados ou importados do sistema de gestão para o sistema de custos. 0 sistema de custos foi preparado para efetuar a distribuição dos custos indiretos aos centros, a distribuição dos custos auxiliares e administrativos e os cálculos de custo unitário, além da montagem dos relatórios gerenciais.

$\mathrm{Na}$ etapa 10 verificou-se se o custo total do hospital havia sido corretamente distribuído para os centros de custos produtivos. Também avaliou-se a incidência dos itens de custos nos centros de custo, computando se houve lançamento exorbitante para determinado centro de custo e se faltava algum lançamento evidente em algum outro centro. 
Quadro 2. Centros de custos.

\begin{tabular}{|c|c|c|c|}
\hline Descrição & Tipo & Descrição & Tipo \\
\hline Unidade de internação - $3^{\circ}$ andar & Produtivo & Serviço de arquivo médico e estatístico & Auxiliar \\
\hline Unidade de internação - $2^{\circ}$ andar & Produtivo & Supervisão de enfermagem & Auxiliar \\
\hline Centro de terapia intensiva & Produtivo & Manutenção & Auxiliar \\
\hline Unidade coronariana & Produtivo & Usina de $\mathrm{O}_{2}$ & Auxiliar \\
\hline Centro cirúrgico & Produtivo & Gerador & Auxiliar \\
\hline Pronto socorro & Produtivo & Central de abastecimento de água & Auxiliar \\
\hline Procardio 1 & Produtivo & Serviço de controle de infecção hospitalar & Auxiliar \\
\hline Procardio 11 & Produtivo & Recepção internação & Auxiliar \\
\hline Hemodinâmica & Produtivo & Recepção pronto socorro & Auxiliar \\
\hline SADT - Raio-X & Produtivo & Recepção exames & Auxiliar \\
\hline SADT - Tomografia & Produtivo & Recepção hemodinâmica e tomografia & Auxiliar \\
\hline SADT - Ultrassonografia & Produtivo & Recepção raio-X & Auxiliar \\
\hline SADT - ECG & Produtivo & Agendamento & Auxiliar \\
\hline SADT - Espirometria & Produtivo & Transporte & Auxiliar \\
\hline SADT - Holter & Produtivo & Segurança & Auxiliar \\
\hline SADT - Mapa & Produtivo & Áreas comuns & Auxiliar \\
\hline SADT - Teste ergométrico & Produtivo & Administração & Administrativo \\
\hline SADT - Eco transtorácico & Produtivo & Comunicação e marketing & Administrativo \\
\hline SADT - Eco transesofágico & Produtivo & Controladoria & Administrativo \\
\hline SADT - Eco de estresse & Produtivo & Financeiro & Administrativo \\
\hline SADT - Laboratório de análises clínicas & Produtivo & Patrimônio & Administrativo \\
\hline SADT - Agência transfusional & Produtivo & Tecnologia da informação & Administrativo \\
\hline Fisioterapia & Produtivo & Departamento pessoal & Administrativo \\
\hline Almoxarifado & Auxiliar & Recursos humanos & Administrativo \\
\hline Farmácia & Auxiliar & Segurança e medicina do trabalho & Administrativo \\
\hline Central de materiais esterilizados & Auxiliar & Faturamento & Administrativo \\
\hline Serviço de nutrição e dietética - SND & Auxiliar & Recursos de glosas & Administrativo \\
\hline Serviço de processamento de roupas & Auxiliar & Compras & Administrativo \\
\hline Serviço de higiene e limpeza & Auxiliar & Telefonia & Administrativo \\
\hline Governança & Auxiliar & Centro de estudos & Administrativo \\
\hline
\end{tabular}

Na etapa 11 foram elaborados os relatórios gerenciais que propiciam informações sobre cada apuração de custo no hospital. Os mais importantes são: relatório de composição, evolução e variação dos custos, relatório de demonstração do custo unitário dos centros de custos produtivos e relatório individualizado por centro de custo.

A Tabela 1 apresenta um modelo de relatório de composição, evolução e variação dos custos ao longo dos períodos. Esse relatório apresenta o custo total do hospital, que é o somatório dos custos diretos e indiretos de todos os centros de custos auxiliares, administrativos e produtivos, agrupados por grupos de itens de custos (pessoal, honorários médicos, materiais, custos gerais e custos indiretos) para os meses de maio, junho, julho e agosto de 2009. Caso seja necessário, é possível analisar quais itens de custo formam o grupo de pessoal ou o grupo de materiais, custos gerais ou custos indiretos. Nesse exemplo, o relatório foi consolidado.

Através desse relatório é possível inferir que o grupo de maior representatividade no hospital é o de materiais, seguido pelo grupo de pessoal, ou seja, esses dois grupos de custos contemplam a maior parcela de custos do hospital.

Outros relatórios podem ser elaborados para a análise de custos, como um relatório por item de custo demonstrando a sua alocação aos centros de custos e a variação entre o mês atual e o anterior e um relatório individualizado por centro de custos produtivo, auxiliar ou administrativo, onde as informações estariam segregadas por grupo de custo e item de custo e possibilitariam observar a incidência e a variação ocorrida em cada item de custo e na própria produção, pois esta pode acarretar aumento ou diminuição no custo unitário do centro de custo.

A Tabela 2 apresenta um relatório que contém o custo total e unitário de cada centro de custos produtivo, possibilitando a análise de dados referente a número de funcionários, custos com pessoal, custos com materiais, custos gerais e indiretos, rateios recebidos, quantidade produzida e, por fim, o custo total. Dividindo-se o custo total do centro de custos produtivo pela quantidade produzida obtêm-se o 
custo médio unitário de cada produção realizada no centro de custos. Ressalta-se que por serem muitos centros de custos produtivos, como ilustração são apresentados apenas cinco centros. Porém a última linha da tabela (total geral) apresenta o custo total de todos os centros e não apenas dos cinco.

Quadro 3. Informações adicionais.

\begin{tabular}{|c|c|}
\hline Centro de custos & Informação \\
\hline Centro cirúrgico & Tempo de cirurgia por paciente \\
\hline Central de materiais esterilizados & Número de itens esterilizados $\mathrm{x}$ peso \\
\hline Serviço de nutrição e dietética & Número de refeições $\mathrm{x}$ peso \\
\hline Serviço de processamento de roupas & $\mathrm{Kg}$ de roupas lavadas \\
\hline Serviço de higiene e limpeza & Área $\left(\mathrm{m}^{2}\right)$ x níveis de criticidade \\
\hline Governança & Minutos trabalhados da governança \\
\hline Serviço de arquivo médico e estatístico & Número de documentos movimentados, arquivados \\
\hline Supervisão de enfermagem & Número de funcionários da enfermagem \\
\hline Manutenção & Minutos trabalhados da manutenção \\
\hline Usina de $\mathrm{O}_{2}$ & $\%$ de consumo de gases medicinais \\
\hline Gerador & $\%$ de consumo do gerador \\
\hline Central de abastecimento de água & \% de consumo da central de abastecimento de água \\
\hline Serviço de controle de infecção hospitalar & Minutos trabalhados do $\mathrm{SClH}$ \\
\hline Recepção internação & Número de internações/transferências \\
\hline Recepção pronto socorro & $100 \%$ pronto socorro \\
\hline Recepção exames & Número de exames da recepção de exames \\
\hline Recepção hemodinâmica e tomografia & $N^{\circ}$ de exames da tomografia e hemodinâmica \\
\hline Recepção raio-X & $100 \%$ raio-X \\
\hline Agendamento & Número de agendamentos \\
\hline Transporte & $\mathrm{Km}$ rodados \\
\hline Segurança & Área $\left(\mathrm{m}^{2}\right)$ - Clínica \\
\hline Áreas comuns & Área $\left(\mathrm{m}^{2}\right)$ - Clínica \\
\hline Administração & Número de funcionários \\
\hline Comunicação e marketing & $\%$ de custos diretos \\
\hline Controladoria & $\%$ de custos diretos \\
\hline Financeiro & $\%$ de custos diretos \\
\hline Patrimônio & Número de itens patrimoniados \\
\hline Tecnologia da informação & Número de equipamentos de informática \\
\hline Departamento pessoal & Número de funcionários \\
\hline Recursos humanos & Número de funcionários \\
\hline Segurança e medicina do trabalho & Número de funcionários \\
\hline Faturamento & Número de itens faturados - Clínica \\
\hline Recursos de glosas & Número de itens glosados \\
\hline Compras & Número de itens solicitados a compras \\
\hline Telefonia & Valor do tarifador \\
\hline Centro de estudos & Número de pacientes-dia, atendimentos e consultas \\
\hline
\end{tabular}

Tabela 1. Relatório de composição, evolução e variação dos custos.

\begin{tabular}{|c|c|c|c|c|c|c|c|c|c|}
\hline \multirow[b]{2}{*}{ Custos } & \multicolumn{3}{|c|}{ Maio/09 } & \multicolumn{3}{|c|}{ Junho/09 } & \multicolumn{3}{|c|}{ Julho/09 } \\
\hline & Valor & $\%$ & $\begin{array}{c}\text { Variação } \\
(\%)\end{array}$ & Valor & $\%$ & $\begin{array}{c}\text { Variação } \\
(\%)\end{array}$ & Valor & $\%$ & $\begin{array}{c}\text { Variaçãc } \\
(\%)\end{array}$ \\
\hline \multicolumn{10}{|l|}{ Custos diretos } \\
\hline pessoal & $691.751,01$ & 31,20 & - & $705.085,26$ & 31,31 & 1,93 & $731.121,29$ & 34,38 & 3,69 \\
\hline Honorários médicos & $331.642,12$ & 14,96 & - & $294.741,92$ & 13,09 & $-11,13$ & $331.105,00$ & 15,57 & 12,34 \\
\hline Materiais & $826.227,37$ & 37,27 & - & $900.015,38$ & 39,96 & 8,93 & $722.591,03$ & 33,98 & $-19,71$ \\
\hline Custos gerais & $272.844,75$ & 12,31 & - & $261.737,01$ & 11,62 & $-4,07$ & $261.727,55$ & 12,31 & 0,00 \\
\hline Total diretos & $2.122 .465,25$ & 95,74 & - & $2.161 .579,57$ & 95,98 & 1,84 & $2.046 .544,87$ & 96,23 & $-5,32$ \\
\hline Custos indiretos & $94.388,38$ & 4,26 & - & $90.521,00$ & 4,02 & $-4,10$ & $80.104,89$ & 3,77 & $-11,51$ \\
\hline $\begin{array}{c}\text { Total } \\
\text { diretos }+ \text { indiretos }\end{array}$ & $2.216 .853,63$ & 100,00 & - & $2.252 .100,57$ & 100,00 & 1,59 & $2.126 .649,76$ & 100,00 & $-5,57$ \\
\hline
\end{tabular}




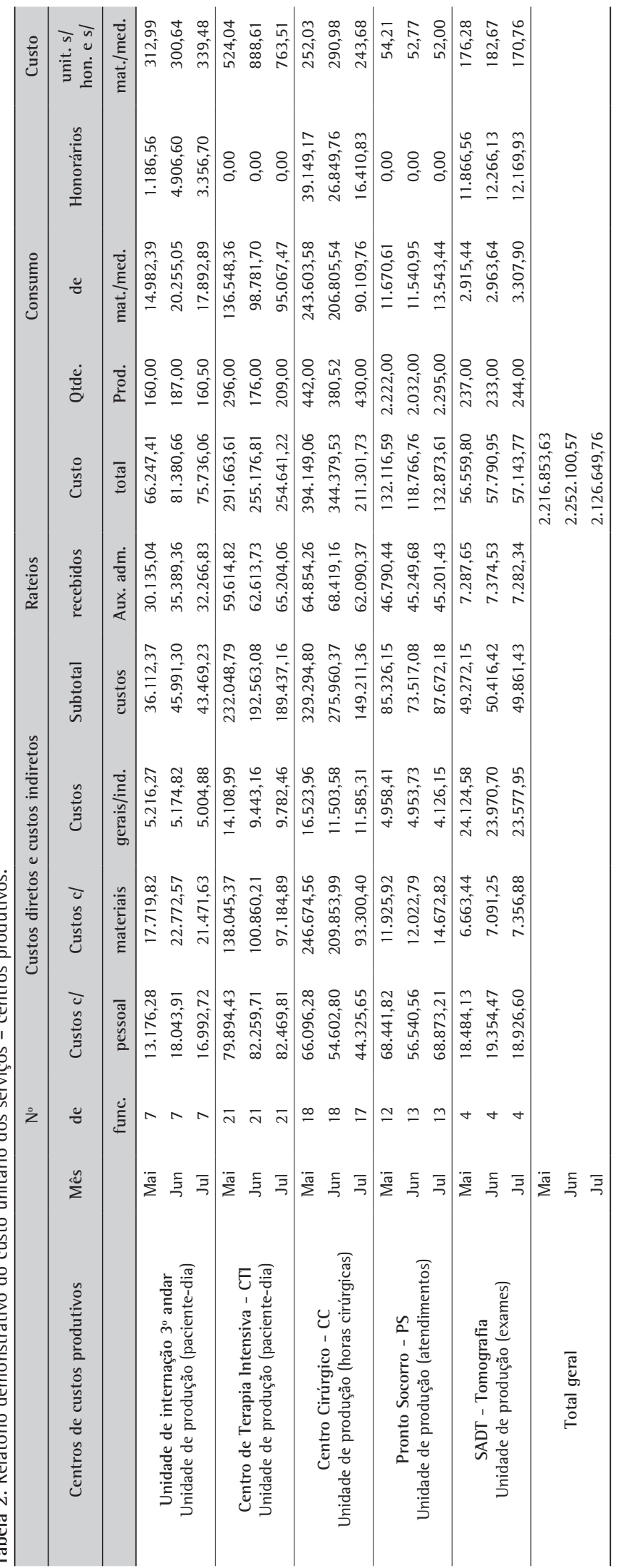


Na etapa 12 foi feita a apuração do custo por procedimento médico-hospitalar com o auxílio das informações do sistema de gestão hospitalar e do sistema de custos. A incidência de cada item da conta do paciente multiplicado pelo custo do respectivo item forma o custo do procedimento. Os itens são lançados na conta do paciente assim que o serviço é realizado. Se o serviço é uma diária logo que o paciente utiliza essa diária a mesma é lançada em sua conta. Se o paciente consome um determinado medicamento, esse é lançado em sua conta e assim por diante. Outra observação importante é: no momento em que o paciente entra na sala cirúrgica inicia-se a medição com a anotação do horário de entrada na sala cirúrgica. Após a utilização da sala cirúrgica é feita a anotação do horário de término da utilização da sala. Portanto, na descrição do item Taxa de sala porte 2, a quantidade refere-se aos minutos utilizados. A Tabela 3 apresenta um modelo de relatório da conta do paciente. Considerando que o mesmo é

Tabela 3. Conta do paciente.

\begin{tabular}{|c|c|c|c|c|c|c|c|}
\hline \multicolumn{8}{|c|}{ Dados do paciente } \\
\hline \multicolumn{4}{|c|}{ Atendimento: 0906005439} & \multicolumn{4}{|c|}{ Tipo de acomodação: Apartamento } \\
\hline \multicolumn{4}{|c|}{ Convênio: Boa Saúde S/A } & \multicolumn{4}{|c|}{ Código procedimento principal: 31009115 Herniorrafia } \\
\hline \multicolumn{4}{|c|}{ Plano: Cobertura total } & \multicolumn{4}{|c|}{ Entrada: 20/06/2009 06h39 Saída: 24/06/2009 09h50 } \\
\hline \multicolumn{4}{|c|}{ Médico: Fulano de tal } & \multicolumn{4}{|c|}{ Especialidade: Cirurgia geral } \\
\hline \multicolumn{8}{|c|}{ Grupo faturamento: materiais e medicamentos } \\
\hline Data & Descrição & Quantidade & $\begin{array}{l}\text { Preço unitário } \\
\text { (R\$) }\end{array}$ & $\begin{array}{l}\text { Preço total } \\
\text { (R\$) }\end{array}$ & $\begin{array}{l}\text { Custo unitário } \\
\text { (R\$) }\end{array}$ & $\begin{array}{l}\text { Custo total } \\
\text { (R\$) }\end{array}$ & $\begin{array}{l}\text { Resultado } \\
\text { (R\$) }\end{array}$ \\
\hline 20/06/09 & Abocath $n^{\circ} 20$ & 1 & 3,90 & 3,90 & 1,76 & 1,76 & 2,14 \\
\hline 20/06/09 & Água destilada $10 \mathrm{~mL}$ & 8 & 0,64 & 5,12 & 0,09 & 0,68 & 4,44 \\
\hline 20/06/09 & Agulha descartável $25 \times 7$ mm & 1 & 0,16 & 0,16 & 0,04 & 0,04 & 0,12 \\
\hline $22 / 06 / 09$ & Equipo Intrafix 1L (Soro) & 2 & 3,02 & 6,04 & 1,38 & 2,76 & 3,28 \\
\hline 20/06/09 & Extensor/perfusor ST20 $120 \mathrm{~cm}$ & 2 & 3,57 & 7,14 & 2,50 & 5,00 & 2,14 \\
\hline 20/06/09 & Fentanest $0,1 \mathrm{mg} / 2 \mathrm{~mL}$ & 2 & 4,41 & 8,82 & 0,63 & 1,26 & 7,56 \\
\hline 20/06/09 & Polifix 2 vias & 2 & 2,76 & 5,52 & 1,75 & 3,50 & 2,02 \\
\hline 20/06/09 & Prolene $2-0$ AG $1 / 226 \mathrm{~mm} 75 \mathrm{~cm}$ & 1 & 10,00 & 10,00 & 6,08 & 6,08 & 3,92 \\
\hline 20/06/09 & Rapifen 2,72 mg/5 mL AMP & 1 & 29,16 & 29,16 & 11,00 & 11,00 & 18,16 \\
\hline $21 / 06 / 09$ & Seringa descartável $3 \mathrm{~mL}$ & 3 & 0,21 & 0,63 & 0,12 & 0,36 & 0,27 \\
\hline $22 / 06 / 09$ & SF 0,9\% Sistema Fechado $100 \mathrm{~mL}$ & 3 & 6,89 & 20,67 & 1,64 & 4,92 & 15,75 \\
\hline $21 / 06 / 09$ & Tilatil 20 mg FA & 3 & 8,06 & 24,18 & 4,76 & 14,28 & 9,90 \\
\hline 20/06/09 & Toradol 30 mg AMP & 1 & 7,00 & 7,00 & 4,59 & 4,59 & 2,41 \\
\hline 20/06/09 & Vicryl 2-0 AG3/8 $31 \mathrm{~mm} 70 \mathrm{~cm}$ & 1 & 15,00 & 15,00 & 9,20 & 9,20 & 5,80 \\
\hline \multicolumn{2}{|c|}{$\begin{array}{l}\text { Total grupo de faturamento: materiais e } \\
\text { medicamentos }\end{array}$} & 375 & & $2.180,13$ & & 932,69 & $1.247,44$ \\
\hline \multicolumn{8}{|c|}{ Grupo faturamento: taxas e diárias } \\
\hline Data & Descrição & Quantidade & $\begin{array}{l}\text { Preço unitário } \\
\text { (R\$) }\end{array}$ & $\begin{array}{l}\text { Preço total } \\
\quad(\mathrm{R} \$)\end{array}$ & $\begin{array}{l}\text { Custo unitário } \\
\text { (R\$) }\end{array}$ & $\begin{array}{l}\text { Custo total } \\
\text { (R\$) }\end{array}$ & $\begin{array}{l}\text { Resultado } \\
\quad \text { (R\$) }\end{array}$ \\
\hline 20/06/09 & Oxigênio Respirador c/c (minutos) & 15 & 0,21 & 3,15 & 0,00 & 0,00 & 3,15 \\
\hline 20/06/09 & Oxigênio Respirador c/c (horas) & 3 & 13,00 & 39,00 & 0,00 & 0,00 & 39,00 \\
\hline 20/06/09 & Dióxido de carbono (uso) & 1 & 25,00 & 25,00 & 0,00 & 0,00 & 25,00 \\
\hline 20/06/09 & 0xigênio em cateter (minutos) & 10 & 0,16 & 1,60 & 0,00 & 0,00 & 1,60 \\
\hline 20/06/09 & Oxigênio em cateter (horas) & 2 & 11,00 & 22,00 & 0,00 & 0,00 & 22,00 \\
\hline 20/06/09 & Tricotomia & 1 & 13,00 & 13,00 & 0,00 & 0,00 & 13,00 \\
\hline 20/06/09 & Aspiração traqueal & 1 & 14,00 & 14,00 & 0,00 & 0,00 & 14,00 \\
\hline 20/06/09 & Taxa controle de uso material & 1 & 87,00 & 87,00 & 0,00 & 0,00 & 87,00 \\
\hline 20/06/09 & Taxa de sala porte 2 & 195 & 230,00 & 230,00 & 4,85 & 945,75 & $-715,75$ \\
\hline 20/06/09 & Bisturi elétrico & 1 & 31,00 & 31,00 & 0,00 & 0,00 & 31,00 \\
\hline 20/06/09 & Taxa sala recuperação pós-anestésica & 1 & 61,13 & 61,13 & 0,00 & 0,00 & 61,13 \\
\hline 20/06/09 & Sondagem retal & 1 & 44,00 & 44,00 & 0,00 & 0,00 & 44,00 \\
\hline 24/06/09 & Diária de enfermaria & 4 & 104,00 & 416,00 & 300,64 & $1.202,56$ & $-786,56$ \\
\hline \multicolumn{2}{|c|}{$\begin{array}{l}\text { Total grupo de faturamento: taxas e diárias - UI } \\
3^{\circ} \text { andar }\end{array}$} & 236 & & 986,88 & & $2.148,13$ & $-1.161,43$ \\
\hline \multicolumn{4}{|c|}{ Resumo geral dos grupos de faturamento } & $\begin{array}{l}\text { Preço total } \\
\text { (R\$) }\end{array}$ & & $\begin{array}{l}\text { Custo total } \\
\text { (R\$) }\end{array}$ & $\begin{array}{l}\text { Resultado } \\
\text { (R\$) }\end{array}$ \\
\hline \multicolumn{3}{|c|}{ Material/medicamento } & & $2.180,13$ & & 932,69 & $1.247,44$ \\
\hline \multicolumn{3}{|c|}{ Taxas/diárias } & & 986,88 & & $2.148,31$ & $-1.161,43$ \\
\hline \multicolumn{3}{|c|}{ Total geral } & & $3.167,01$ & & $3.081,00$ & 86,01 \\
\hline
\end{tabular}


muito extenso, alguns itens foram excluídos para facilitar a apresentação. Por isso, alguns itens foram excluídos da conta, o que fará com que o valor total do grupo de faturamento materiais e medicamentos e do grupo de faturamento taxas e diárias não seja a somatória dos itens descritos acima.

$\mathrm{Na}$ Tabela 3 existem alguns itens com custo zerado. lsto ocorre porque o item gera receita, pois tratam-se de itens passíveis de cobrança. Porém tais itens não têm seu custo identificado pelo sistema de custos, estando o mesmo agregado ao custo do setor.

Por fim, na etapa 13 foi feita a análise de resultados. Através das etapas 9, 10 e 11 foi possível obter o custo médio por paciente-dia, custo médio da hora cirúrgica, custo médio por exame, dentre outros. 0 custo médio é obtido através da divisão do custo total (subtraindo o custo com materiais e medicamentos e honorários) do centro de custos pelo número de unidades produzidas pelo centro de custos. Uma vez estabelecidos, na etapa 11, os relatórios gerenciais para acompanhamento e análise dos custos pelo responsável pelo gerenciamento de cada área, essas informações de custos dos períodos, comparadas com os meses anteriores, são indicadores importantes tanto no gerenciamento dos custos e avaliação da produtividade como na comparação com os valores de receita obtida. Os hospitais podem ter natureza diferente (SUS, convênios, particulares), e para cada natureza existe uma regra de remuneração pré-estabelecida sobre a qual incidirá a respectiva receita pelo serviço prestado. Ou seja, além da gestão de custos, é importante que o hospital contemple a análise de resultados. A Tabela 4 é um exemplo de relatório de análise de resultados por centro de custos produtivos que apresenta a receita obtida

Tabela 4. Análise de resultado por centro de custo unidade de internação.

\begin{tabular}{lccc}
\hline \multicolumn{1}{c}{$\begin{array}{c}\text { Unidade de lnternação } \\
3^{\circ} \text { andar }\end{array}$} & Maio & Junho & Julho \\
\hline Receita (R\$) & $64.147,73$ & $51.410,20$ & $78.932,91$ \\
Custo variável (R\$) & $20.588,23$ & $16.673,18$ & $26.926,04$ \\
Custo com Mat./med. (R\$) & $12.708,01$ & $11.343,34$ & $19.029,14$ \\
Margem de contribuição (R\$) & $43.559,50$ & $34.737,02$ & $52.006,87$ \\
Margem de contribuição (\%) & $68 \%$ & $68 \%$ & $66 \%$ \\
Custo fixo (R\$) & $45.659,18$ & $64.707,47$ & $48.810,02$ \\
Resultado (R\$) & $-2.099,68$ & $-29.970,45$ & $3.196,85$ \\
RESULTAD0 (\%) & $-1 \%$ & $-11 \%$ & $9 \%$ \\
Custo unitário & 312,99 & 300,64 & 339,48 \\
(sem mat./med., em R\$) & 7 & 7 & 7 \\
№ funcionários & 160 & 187 & 160 \\
№ de pacientes-dia & 7 & 7 & 7 \\
No leitos & 31 & 30 & 31 \\
Dias mês & 73,73 & 89,50 & 73,73 \\
Taxa de ocupação - \% & 168 & 348 & 150 \\
Ponto de equilíbrio & & & \\
\hline
\end{tabular}

pela remuneração dos serviços prestados. Dela são diminuídos os custos variáveis para se chegar ao valor da margem de contribuição para a cobertura dos custos fixos e representa, nos meses de maio, junho e julho, cerca de 68\%, 68\% e 66\%, respectivamente, da receita. 0 relatório traz também informações quanto ao resultado do centro de custos, ou seja, o lucro obtido nos períodos e o ponto de equilíbrio, ou seja, o centro de custos precisa atender 168 , 348 e 150 pacientes-dia nos referidos meses para cobrir custos fixos e variáveis. Outra informação que merece destaque e pode ser útil para a tomada de decisão é a taxa de ocupação, que está em 73,73\%, $89,50 \%$ e $73,73 \%$, respectivamente para os meses de maio, junho e julho. Esse modelo de relatório pode ser elaborado para os demais centros de custos produtivos do hospital.

A Tabela 5 apresenta um relatório para análise dos procedimentos realizados no hospital. Na primeira coluna listam-se os procedimentos realizados no hospital no mês de junho de 2009, seguidos pela receita total. Na terceira coluna listam-se os custos variáveis (custos com materiais e medicamentos) e a partir deste é possível obter a margem de contribuição. Em termos percentuais, a margem de contribuição representa a parcela com que cada unidade monetária obtida com a venda dos serviços contribui para cobrir os custos fixos ou para formar o lucro. Esses conceitos contribuem para o planejamento de estratégias e para a tomada de decisões em geral.

Com o uso desse relatório (Tabela 5) é possível analisar qual procedimento apresenta melhor resultado para o hospital e também qual apresenta resultado insatisfatório. Esta análise contempla o valor faturado (receita) pelo serviço prestado e apresenta também a separação dos custos em fixos (custos com serviços) e variáveis (custos com materiais e medicamentos), possibilitando, com isso, obter a margem de contribuição de cada procedimento. Como ilustração, a Tabela 5 apresenta 20 tipos de procedimentos do hospital, de um total de 502 tipos de procedimentos realizados no mês de junho.

Ressalta-se que quando um determinado procedimento é repetitivo, o valor deste é consolidado em uma única linha. Por exemplo, se existem cinco colecistectomias sem colangiografia realizadas neste mês, as cinco são consolidadas em uma única linha. Logo, para saber o valor de cada uma é necessário analisar conta por conta. Nesse caso justifica-se a análise considerando a média dos valores apresentados para o respectivo procedimento.

A determinação do custo por procedimento (que pode ser agrupado por médico, por especialidade e por convênio) é importante, porque afetará um grande número de análises e ações a serem tomadas 


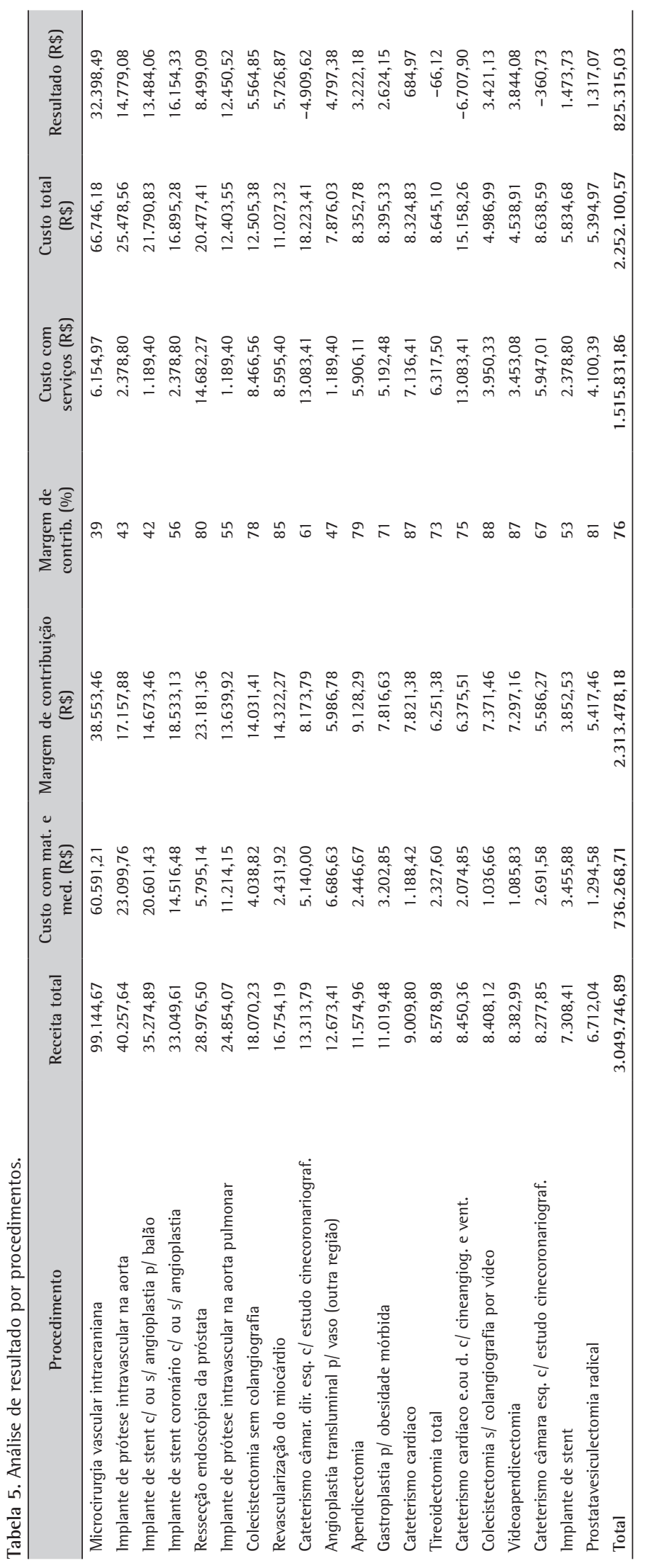


no hospital. Uma correta determinação do custo do procedimento servirá de parâmetro para traçar as estratégias de gestão de custos: Estabelecer o preço médio de cada procedimento médico; Estimar os custos para negociação por pacote (constitui a padronização do tempo de internação, utilização da sala cirúrgica, exames, materiais e medicamentos, dentre outros itens para cada tipo procedimento médico-hospitalar); Determinar as margens brutas associadas às especialidades e procedimentos médicos; Estimar a rentabilidade das especialidades e procedimentos médicos; e Confrontar o valor faturado com o custo do procedimento médico.

Um hospital é uma empresa complexa interligada por processos representados aqui em vários centros de custos que oferecem serviços ao paciente, e cada um desses centros irá refletir no produto final - o procedimento, portanto é importante evoluir da análise por centros de custos para a análise de procedimento.

\section{Conclusão}

A implantação de um sistema de custos em instituições de saúde, além de propiciar a gestão de custos, deve também auxiliar a análise de resultados. Fazer a gestão da saúde é tão complexo quanto fazer a gestão de outras áreas. Tão importante quanto fazer a gestão de custos é analisar os resultados e confrontar custo com a respectiva receita. Para isso é necessário, primeiramente, a estruturação de um sistema de custos.

0 ABC é visto por alguns autores (MARTINS, 2000; CHING, 2001) como o método de custeio mais apropriado para hospitais. Entretanto, sua implantação completa em instituições de saúde, em especial hospitais, é complexa e demanda muito esforço. Sua manutenção envolve a coleta de muitas informações, encarecendo também o sistema de custeio. Assim, a viabilidade prática da utilização efetiva do $A B C$ em todas as unidades de um hospital é pequena. 0 $A B C$ pode auxiliar os gestores se aplicado em uma unidade específica para melhorar o processo. Mesmo o método dos centros de custos, mais simples do que o $A B C$, exige esforço significativo para ser implantado em hospitais por causa da estrutura complexa. Por isto, entende-se que o método dos centros de custos ainda é o mais adequado para gerar informações de custos para a organização hospitalar como um todo.

0 custo dos serviços, ou seja, dos centros de custos produtivos, apurados pelo método dos centros de custos, disponibiliza dados necessários que, com os demais dados, são lançados na conta do paciente (custos diretos; material e medicamentos; e outros lançamentos feitos pelo faturamento), para que seja possível a apuração do custo por procedimento, sendo esse o objetivo final do ciclo da apropriação dos custos, uma vez que o hospital é remunerado pelos procedimentos. Assim, o custo por procedimento médico-hospitalar fornece informação estratégica para a tomada de decisão.

Porém, para que se obtenha uma apuração de custos acurada faz-se necessário, além do já mencionado, que todos os setores envolvidos no processo de apuração enviem as informações pertinentes para o setor de custos.

A sistemática para apuração dos custos por procedimento médico-hospitalar apresentada contribui para a melhoria do gerenciamento operacional de instituições de saúde. A aplicação da mesma em um hospital apresentou bons resultados, proporcionou informações importantes que normalmente não estão disponíveis para os gestores do hospital e, enfim, possibilitou informações confiáveis que auxiliam na tomada de decisões.

Para implantação da sistemática proposta é importante o apoio da direção da instituição de saúde, pois a resistência dos setores à implantação de controles é sempre uma barreira delicada e difícil de transpor. 0 pessoal envolvido no processo deve estar consciente de que o principal objetivo do trabalho é melhorar o gerenciamento da instituição. Deve-se destacar a necessidade de controles para a efetiva aplicação do sistema, mas os mesmos não são o objetivo final. Ressalta-se que, com base nas informações geradas pela sistemática, a administração é quem deve decidir sobre as ações que precisam ser tomadas para melhorar os resultados.

\section{Referências}

ABBAS, K. Gestão de custos em organizações hospitalares. 2001. Dissertação (Mestrado em Engenharia de Produção)-Universidade Federal de Santa Catarina, Florianópolis, 2001.

BAUMGARTNER, R. R. Avaliação da aplicabilidade do custeio $A B C$ - activity based costing na acurácia de custos na área hospitalar, especificamente na Unidade de Terapia Intensiva: Estudo de um caso prático. 1998. Dissertação (Mestrado em Ciências Financeiras e Contábeis)-Pontifícia Universidade Católica de São Paulo, São Paulo, 1998.

BEULKE, R.; BERTÓ, D. J. Gestão de Custos e Resultado na Saúde. São Paulo: Saraiva, 1997.

BORNIA, A. C. Análise gerencial de custos: uma aplicação em empresas modernas. 2. ed. São Paulo: Atlas, 2009.

BRUGER, N. et al. Financial management: concepts and applications for health care organizations. Dubuque: Kendall, Hunt, 1997.

CHING, H. Y. Manual de custos de instituições de saúde: sistemas tradicionais de custos e sistema de custeio baseado em atividades. São Paulo: Atlas, 2001. 
COLTRO, A.; SANTOS, S. A. A busca da compreensão da racionalidade e da ética da ação administrativa na gestão de uma organização hospitalar pública. In: ENCONTRO DA ASSOCIAÇÃO NACIONAL DE PÓS-GRADUAÇÃO E PESQUISA EM ADMINISTRAÇÃO - ANPAD, 22., 1998, Foz do lguaçu. Anais eletrônicos... Foz do lguaçu, 1998.

FALK, J. A. Gestão de custos para hospitais. São Paulo. Atlas. 2001.

MAHER, M. Contabilidade de custos: criando valor para a administração. Tradução José Evaristo dos Santos. São Paulo: Atlas, 2001.

MARTINS, D. Custos e orçamentos hospitalares. São Paulo: Atlas, 2000.

MARTINS, V. F. Desenvolvimento de um modelo de resultados em serviços hospitalares com base na comparação entre receitas e custos das atividades associadas aos serviços. 2002. Dissertação (Mestrado em Engenharia de Produção)-Universidade Federal de Santa Catarina, Florianópolis, 2002.

MATOS, A. J. Gestão de custos hospitalares: técnicas, análise e tomada de decisão. São Paulo: Editora STS, 2002.

OLIVEIRA, A. D. S. et al. Estratégia, custos e hospitais: um diagnóstico na cidade de Salvador, Bahia (2006). In: CONGRESSO BRASILEIRO DE CUSTOS, 14., 2007, João Pessoa. Anais eletrônicos... João Pessoa, 2007.

PEREZ JUNIOR, J. H.; OLIVEIRA, L. M.; COSTA, R. G. Gestão estratégica de custos. 6. ed. São Paulo: Atlas, 2009.

RAIMUNDINI, S. L. et al. Aplicabilidade do custeio baseado em atividades e análise de custos em hospitais públicos. Revista de Administração, v. 41, n. 4, p. 453-465, out./ dez. 2006.

RIBEIRO, O. S. Contabilidade de custos. São Paulo: Saraiva, 2009.

ROCHA, V. L. Dificuldades na implantação de sistemas de custeio em instituições hospitalares: estudo de caso em um hospital de Florianópolis. 2004. Dissertação
(Mestrado em Engenharia de Produção)-Universidade Federal de Santa Catarina, Florianópolis, 2004.

SANTOS, J. J. Contabilidade e análise de custos: modelo contábil, Métodos de depreciação, ABC - custeio baseado em atividades, análise atualizada de encargos sociais sobre salários. 5. ed. São Paulo: Atlas 2009.

SCHULTZ, C. A. Gestão dos custos de serviços públicos de alta complexidade hospitalar. 2007. Dissertação (Mestrado em Contabilidade)-Universidade Federal de Santa Catarina, Florianópolis, 2007.

SILVA, E. L.; MENEZES, E. M. Metodologia da pesquisa e elaboração de dissertação. 3. ed. rev. atual. Florianópolis: Laboratório de Ensino a Distância da UFSC, 2001.

SILVA, M. Z. Mensuração dos custos de procedimentos médicos em organizações hospitalares: sistematização de um método de custeio híbrido à luz do ABC e da UEP. 2006. Dissertação (Mestrado em Contabilidade)-Universidade Federal de Santa Catarina, Florianópolis, 2006.

SOUZA, P. C. F. Como são apurados os custos dos procedimentos médicos: o caso do IMIP - Instituto Materno Infantil de Pernambuco. 2004. Dissertação (Mestrado em Administração)-Universidade Federal de Pernambuco, Recife, 2004.

SOUZA, A.; CLEMENTE, A. Gestão de custos: aplicações operacionais e estratégicas: exercícios resolvidos e propostos com utilização do Excel. São Paulo: Atlas, 2007.

SOUZA, A. A. et al. Modelagem do custeio baseado em atividades para o centro cirúrgico de hospitais. In: ENCONTRO NACIONAL DE ENGENHARIA DE PRODUÇÃo, 28., 2008, Rio de Janeiro. Anais eletrônicos... Rio de Janeiro, 2008.

VARGAS, O. C. O custeio baseado em atividades aplicado em serviços de UTI hospitalar. 2002. Dissertação (Mestrado em Engenharia de Produção)-Universidade Federal de Santa Catarina, Florianópolis, 2002.

\title{
Systematic for determination of hospital costs per medical procedure hospital
}

\begin{abstract}
The aim of this paper is to present a systematic investigation of hospital costs per medical procedure and thus contribute to improving the management of the operating results of health institutions. Research costs in health care usually have focused on the choice and application of methods of costing (ABC, cost centers), without giving emphasis to the generation of data for decision making. However, the deployment of a cost system in health institutions, in addition to providing cost management, should also assist in the analysis of results. Research can be classified as applied, exploratory and qualitative use of action research. We proposed a systematic comprised of thirteen sequential stages allowing the hospital to verification of the result by hospital medical procedure. It was concluded that the use of systematic information available to the hospital that allow the knowledge of the cost per procedure, and other information that contribute to the improvement of performance management.
\end{abstract}

Keywords

Method of costs centers. Analysis of results. Analysis procedures. Cost-volume-profit. Hospital costs. 